\title{
Interfacing dielectric elastomer actuators with liquids
}

Alexandre Poulin, Luc Maffli, Samuel Rosset and Herbert R. Shea

Proc. SPIE 9430, Electroactive Polymer Actuators and Devices (EAPAD) 2015, 943011 (1 April 2015); doi: $10.1117 / 12.2083837$

Copyright notice format: Copyright 2015 Society of Photo-Optical Instrumentation Engineers. One print or electronic copy may be made for personal use only. Systematic reproduction and distribution, duplication of any material in this paper for a fee or for commercial purposes, or modification of the content of the paper are prohibited. 


\title{
Interfacing dielectric elastomer actuators with liquids
}

\author{
Alexandre Poulin, Luc Maffli, Samuel Rosset and Herbert Shea \\ Microsystems for Space Technologies Laboratory, Ecole Polytechnique Fédérale de Lausanne, \\ Neuchâtel, Switzerland
}

\begin{abstract}
Methods and materials for liquid encapsulation in thin $(19 \mu \mathrm{m})$ silicone membranes are presented in this work. A set of 12 liquids including solvents, oils, silicone pre-polymers and one ionic liquid are experimentally tested. We show that all selected liquids are chemically inert to silicone and that vapor pressure is the key parameter for stable encapsulation. It is demonstrated that encapsulated volume of silicone pre-polymers and ionic liquids can stay stable for more than 1 month.

The actuation of dielectric elastomer actuators (DEAs) in conductive liquids is also investigated. An analysis of the equivalent electrical circuits of immersed DEAs shows that non-overlapping regions of the electrodes should be minimized. It also provides guidelines to determine when the electrodes should be passivated. The effects of immersion in a conductive liquid are assessed by measuring the actuation strain and capacitance over periodic actuation. The experimental results show no sign of liquid-induced degradation over more than 45k actuation cycles.
\end{abstract}

Keywords: Dielectric elastomer actuators, liquids, encapsulation, immersion

\section{INTRODUCTION}

Dielectric elastomer actuators (DEAs) are a class of soft-actuators which can achieve actuation strain greater than $1000 \%{ }^{1}$ and sub-millisecond response time ${ }^{2}$. They consist of a dielectric elastomer membrane sandwiched between two stretchable electrodes. When a voltage difference is applied across the device, electrostatic forces induce Maxwell stresses across the membrane. As a result, the incompressible elastomer undergoes an out-of-plane compression and an in-plane elongation. Many promising applications of dielectric elastomer actuators (DEAs) require direct contact with liquids. In some cases they are designed to specifically act on the liquid. For example, micro-pumps ${ }^{3-7}$ and tunable lenses $^{2,8,9}$ generate flow and move an encapsulated volume, respectively. In other cases they are simply designed to operate in a liquid environment. For instance, biomedical applications ${ }^{10,11}$ typically require immersion in a conductive aqueous solution (growth medium). Regardless of the application, interfacing DEAs with liquids remains a challenging task and only little information can be found in literature.

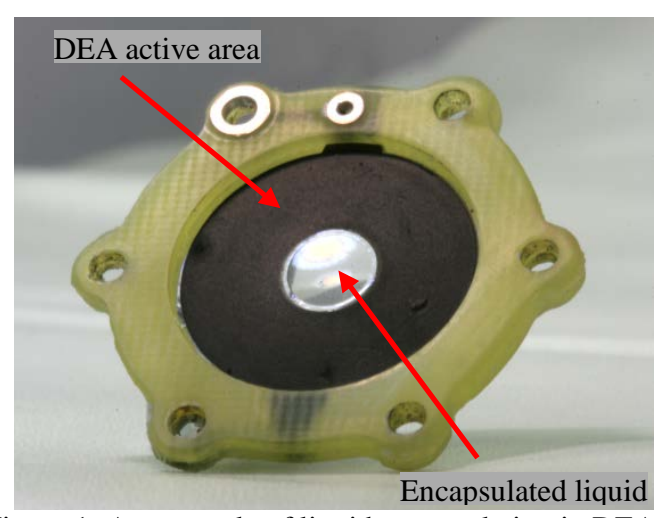

Figure 1: An example of liquid encapsulation in DEAs. In this device, the central encapsulated liquid serves as an optical lens which focal distance can be tuned by actuating the surrounding active area. Good encapsulation is critical since it directly impacts the optical and mechanical performance of the tunable lens.

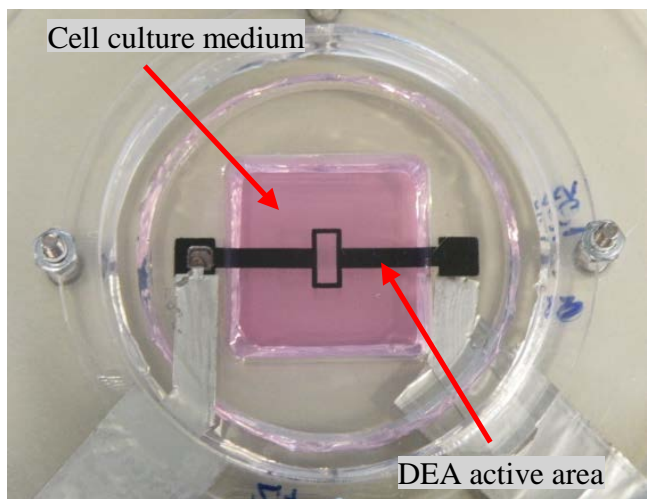

Figure 2: An example of DEA operating in a liquid environment. The device is used to apply periodic mechanical stress on biological cells. For the cells to survive, the actuator must be immersed in cell growth medium (conductive aqueous solution). The liquid should not affect the device life time and stability, both central to successful biological experiments. 
Interfacing DEAs with liquids presents two main difficulties: the encapsulation of liquids in soft elastomer membranes and the operation of DEAs in a liquid environment, examples are respectively presented in Figure 1 and Figure 2. The encapsulated fluids must meet strict requirements in terms of compatibility. Namely, they must be chemically inert to the actuator and show little diffusion through the silicone elastomer membrane. Depending on the application, they might need to exhibit specific optical and mechanical properties such as transparency or low viscosity. A few examples of encapsulation can be found in literature. For instance, two different tunable lenses using $\mathrm{VHB}^{8}$ and silicone ${ }^{2}$ membranes were reported. In both cases the lens is composed of an encapsulated drop of silicone pre-polymer (uncured base). The encapsulation of a proprietary liquid from Cargille was also used the development of a MEMS-based tunable liquid lens ${ }^{12,13}$. Apart from applications in optics, hydrostatically-coupled DEAs were also reported ${ }^{14-16}$. Encapsulation of water, corn oil, silicone grease and talcum powder was studied. Despite the complexity of liquid encapsulation in DEAs, only little and sparse information can be found on the subject. Although most reported works use acrylic elastomers membranes, this work focuses on silicone elastomers which provide much better mechanical performances. The resources for silicones are mainly limited to some reference works on swelling of PDMS (Sylgard 184, Dow Corning) that can be found in microfluidics literature ${ }^{17}$.

Operation of DEAs in a liquid environment also presents interesting challenges. First of all, the immersion liquid is not a design parameter but is rather a constraint given by the application. A few examples of DEA working in a liquid environment can be found in literature such as micro-pumps ${ }^{3-7}$ and deformable cell culture systems ${ }^{10,18}$. For most applications, the immersions liquids are aqueous solutions and therefore conductive media. It is well known that water (or more precisely water vapor) can easily diffuse through silicone. While the resulting loss of liquid is usually not an issue, the potential diffusion of contaminants into the membrane might raise concerns. The accumulation of impurities into the membrane could change its Young's modulus, as well as its electrical permittivity and conductivity, thus altering the actuator performance. In the case of ionic diffusion, the accumulation of charged particles in the membrane can decrease the breakdown voltage and lead to premature failure of the device. Only little information can be found in literature about long term stability of DEAs in conductive liquid environments. Diffusion of ions from the immersion fluid into the actuator membrane has been suggested ${ }^{18}$ but its investigation has never been reported.

In this paper we address the challenges of interfacing DEAs with liquids. A first section presents a study on liquidcompatibility of silicone elastomers. Materials considerations and requirements for fluidically-coupled DEAs are discussed. A list of liquids including polar solvents, oils, silicone pre-polymers and one ionic liquid are selected for investigation. Their compatibility with thin silicone membranes is evaluated based on the results of two experiments: The first test was to dispense a few drops of liquid on an elastomer membrane and to monitor any changes that could be observed by visual inspection. The second test was to encapsulate a few drops of liquid under a silicone membrane and to monitor the height of the resulting bulge. A second section to the article presents a study on the immersion of DEAs in conductive liquids. Design considerations are discussed for single-sided and full immersion. The effects on the actuation behavior and on the equivalent electrical circuit are discussed. In order to assess the effects of immersion, the actuation strain and the capacitance of a DEA are monitored over periodic actuation.

\section{LIQUID ENCAPSULATION IN THIN SILICONE ELASTOMER MEMBRANES}

\subsection{Material considerations and requirements}

Compatibility of liquids with silicone can be evaluated using several parameters. The first and most important one is chemical stability, which, regardless of the application (immersion, transport or encapsulation), needs to be satisfied. Although many liquids are considered to be chemically inert to silicone, water is usually seen as the reference. Depending on the application, additional criteria can be considered. One of them is solubility, which corresponds to the solvent ability to dissolve in silicone. If the application requires liquid encapsulation, such as tunable lenses or hydrostatically-coupled DEAs, the solubility should be as low as possible in order to maintain the encapsulated volume constant. For the same reason, considering the gas permeability of silicone, it is also important for encapsulated liquids to have low vapor pressures. Another criterion to consider is swelling, which corresponds to a volume expansion due to solvent penetration. Swelling can affect both mechanical and electrical properties of silicone and should be avoided. It is usually measured as a ratio, comparing the dimensions before and after a long period ( $>24 \mathrm{~h})$ of immersion in a solvent. Non-polar solvent such as hydrocarbons are known to induce large swelling in silicone, while polar solvents usually have much lower swelling ratios. It is important to understand that the notions of solubility and swelling are not necessarily correlated ${ }^{17}$, and should therefore be considered as two independent evaluation criteria. 
A series of 12 liquids were selected and tested for encapsulation in thin silicone membranes. The liquids were selected based on their material properties, with the objective of providing chemical stability as well as minimal diffusion (solubility) and swelling. In addition to these criteria, only transparent fluids with relatively low viscosity were considered, therefore making the encapsulation process and materials compatible with a broad range of DEAs applications: viscosity affects fluidically-coupled DEAs (effectively limiting their mechanical response time), and optical transparency is essential for DEA-based optical components (ex. tunable lenses). Table 1 presents the selected liquids with their corresponding swelling ratio (for Sylgard 184), vapor pressure and dynamic viscosity. The list includes solvents, silicone pre-polymers, oils and one ionic liquid. The solvents were selected based upon their swelling ratio, choosing only the ones with low or negligible values. As we can see, vapor pressures are not uniform amongst the solvents. Based on this parameter only, ethylene glycol and propylene carbonate are expected to be the most compatible solvents. The silicone pre-polymers were chosen for their negligible vapor pressure. Sylgard 184 has a higher optical transparency while LSR-20 (Factor-II, Inc.) has lower viscosity. Sylgard 184 encapsulation in VHB membranes has already been reported ${ }^{8}$ but their compatibility with silicone hasn't been investigated yet. The oils were selected for their low vapor pressure and low viscosity. The list includes microscope immersion oil (Leica Type F), safflower oil, general purpose lubricant oil (Komet, Fessmann \& Hecker) and vacuum pump oil (Edward Ultra Grade 19). As for the ionic liquid, bis(trifluoromethylsulfonyl)imide $\left(\mathrm{TF}_{2} \mathrm{~N}\right.$ IoLiTec $\left.\mathrm{GmbH}\right)$ was selected for its negligible vapor pressure and low viscosity. In addition, it can be used as a liquid electrode due to its high electrical conductivity ${ }^{19}$.

The electrical properties of the liquids are listed in the last column of Table 1 . At one end of the spectrum we have the silicone pre-polymers and oils which are very good insulators. On the other end, we have the ionic liquid which is highly conductive. As for the solvents, they typically exhibit low conductivity. Electrical properties of the liquids is an important aspect to consider and will be discussed in more details in the next section. One concern is that diffusion of molecules in the membrane can potentially change its dielectric constant and conductivity. Such modifications would directly impact DEAs performance, potentially inducing dielectric breakdown or short-circuit across the electrodes.

Table 1: Compatibility between the listed liquids and thin silicone membranes was tested. The liquids selection was based on the materials swelling ratio of PDMS, vapor pressure, dynamic viscosity and electrical properties. The table is adapted from Maffli's thesis on Fluidically-coupled elastomer actuator structures for tunable optics and microfluidics ${ }^{19}$.

\begin{tabular}{|c|c|c|c|c|c|}
\hline \multicolumn{2}{|r|}{ Liquids } & $\begin{array}{l}\text { Swelling ratio } \\
\text { of Sylgard } 184\end{array}$ & $\begin{array}{c}\text { Vapor pressure } \\
\text { at } \mathbf{3 0 0} \mathbf{K} \\
{[\mathrm{mmHg}]}\end{array}$ & $\begin{array}{c}\text { Dynamic viscosity } \\
\text { at } \mathbf{3 0 0} \mathbf{K} \\
{[\mathrm{Pas}]}\end{array}$ & $\begin{array}{c}\text { Electrical } \\
\text { Conductivity } \\
{\left[\mathrm{Sm}^{-1}\right]}\end{array}$ \\
\hline \multirow{6}{*}{$\begin{array}{l}\frac{n}{2} \\
\frac{2}{0} \\
\text { ज }\end{array}$} & DI water & 1.00 & 26.7 & $8.9 \times 10^{-4}$ & $\sim 10^{-4}$ \\
\hline & Glycerol & 1.00 & $0.2 \times 10^{-3}$ & $9.3 \times 10^{-1}$ & $0.1 \times 10^{-6}$ \\
\hline & Ethylene Glycol & 1.00 & 0.08 & $1.6 \times 10^{-2}$ & $1.07 \times 10^{-6}$ \\
\hline & Dimethylsulfoxide (DMSO) & 1.00 & 0.42 & $2.0 \times 10^{-3}$ & $3 \times 10^{-8}$ \\
\hline & N,N-dimethylformamide (DMF) & 1.02 & 2.7 & $7.9 \times 10^{-4}$ & $2.5 \times 10^{-4}$ \\
\hline & Propylene Carbonate & 1.01 & 0.13 & $2.7 \times 10^{-3}$ & - \\
\hline \multirow{2}{*}{ 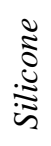 } & LSR20 silicone component B & - & negligible & 3 to 4 & Insulator \\
\hline & Sylgard 184 prepolymer & - & Negligible & 3.9 & Insulator \\
\hline \multirow{4}{*}{$\stackrel{\mathscr{\sigma}}{\tilde{\sigma}}$} & Leica type F microscope oil & - & Low & Medium & Insulator \\
\hline & Safflower oil & - & Low & $2.7 \times 10^{-2}$ & Insulator \\
\hline & Tool lubricant oil & - & $<7.5 \times 10^{-2}$ & $6.07 \times 10^{-2}$ & Insulator \\
\hline & Vacuum pump oil & - & $7.5 \times 10^{-9}$ & $1.23 \times 10^{-1}$ & Insulator \\
\hline & Ionic liquid $\left(\mathrm{TF}_{2} \mathrm{~N}\right)$ & - & Negligible & $3.48 \times 10^{-2}$ & $0.88 \mathrm{Sm}^{-1}$ \\
\hline
\end{tabular}




\subsection{Liquid encapsulation process}

An encapsulation process was developed to test the compatibility of liquid with thin silicone membranes and is presented in Figure 3: (a) The first step is to activate by oxygen plasma a thin silicone membrane and a rigid silicone plate. The thin silicone membrane is made of CF19-2186 (NuSil Technology LLC) or LSR4305 (Bluestar Silicones) and is suspended on a poly(methyl methacrylate) (PMMA) rigid frame. Biaxial pre-stretch values ranging from 1.2 to 1.5 were used. The final thickness (after pre-stretch) was kept constant at $19 \mu \mathrm{m}$. (b) In the second step, the membrane is laid on a CNC-machined vacuum chuck made of a porous material (Metapor, Portec Ltd.). The chuck has a set of identical through-holes (3mm in diameter) where the membrane gets pulled-in when vacuum is applied on the back-side of the chuck. (c) The cavities thus created are used as reservoirs and filled with different liquids. The rigid silicone plate previously activated by oxygen plasma is then placed on top of the membrane, sealing the reservoirs. A small pressure is maintained on top of the assembly for one hour to achieve good bonding and avoid leakage due to membrane delamination. (d) In the final step vacuum is removed, the sample is released and the rigid frame is cut off. Trapped air bubbles are inevitable but diffuse within a few hours through the top silicone membrane.

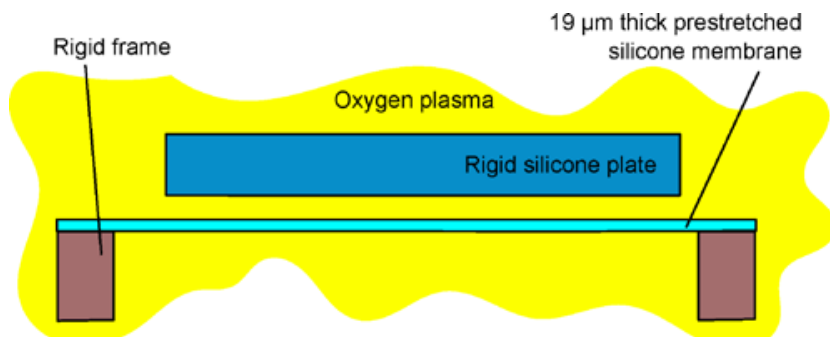

(a) The surfaces of a soft silicone membrane and a rigid silicone plate are activated by oxygen plasma (for subsequent bonding step).

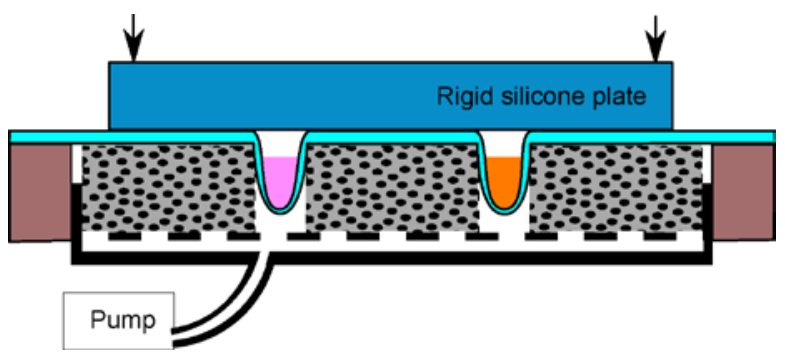

(c) The reservoirs are filled with liquid and sealed with a rigid silicone plate. Pressure is maintained for a few minutes between the two silicone parts to achieve good bonding.

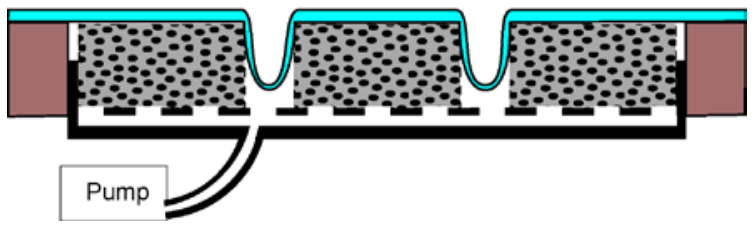

(b) The soft membrane is positioned on top of a porous chuck with four holes. Vacuum is applied to deform the membrane and create reservoirs.

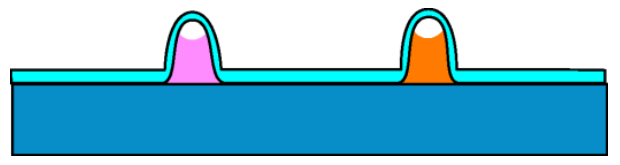

(d) The vacuum is removed and the sample is released. Trapped air bubbles are inevitable but diffuse through the top membrane within a few hours.

Figure 3: Process for encapsulation of liquid droplets in thin silicone elastomer membranes.

\subsection{Experimental results}

A first experiment was used to quickly filter out any incompatible solvents from Table 1. A few drops of solvents were dispensed on top of a $40 \mu \mathrm{m}$ thick suspended silicone membrane (Sylgard 184 with no pre-stretch). Visual inspection showed no impact on the membrane for water, glycerol, ethylene glycol and propylene carbonate. However, DMSO and DMF which were also expected to exhibit good compatibility diffused quickly into silicone. Within less than $30 \mathrm{~s}$ after dispensing, small droplets of solvents started forming on the back side of the suspended membrane. Figure 4 presents the results obtained with DMSO. The blurred region corresponds to the droplet-covered area, which, as we can see, is larger than the initial drop size. Visual inspection indicated not clear sign of swelling but these results clearly demonstrate high solubility into silicone. As a result DMSO and DMF were discarded for the second compatibility test. 


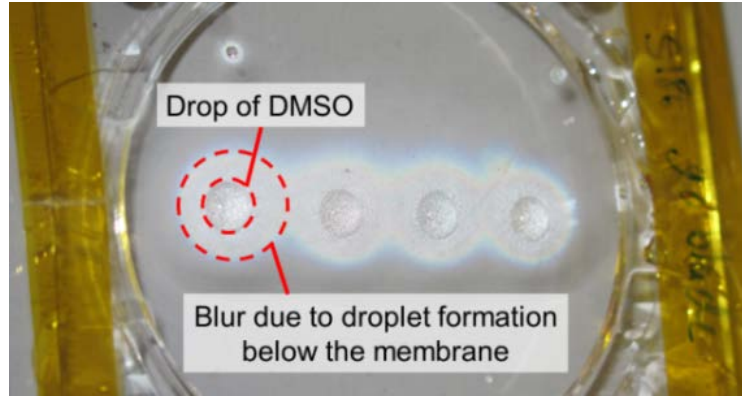

Figure 4: Drops of dimethyl sulfoxide were placed on a $40 \mu \mathrm{m}$ thick silicone membrane. DMSO quickly diffused through the membrane, creating droplets on the back side.

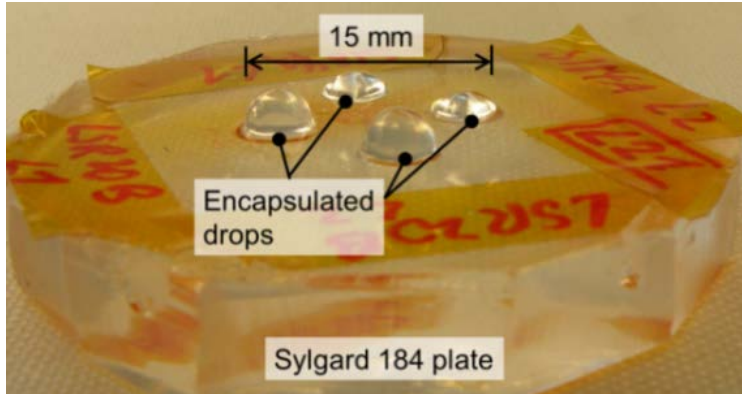

Figure 5: Four different liquids were encapsulated under a $19 \mu \mathrm{m}$ thick silicone membrane. The reservoir initial diameter is $3 \mathrm{~mm}$.

The second experiment was done to compare the performance of remaining liquids over longer periods of time. Using the liquid encapsulation process previously described, $3 \mathrm{~mm}$ diameter liquid drops were encapsulated between a prestretched membrane and a rigid silicone plate. Figure 5 presents an example where four different liquids were individually encapsulated. After the encapsulation process was completed, the height of each encapsulated droplet was monitored using an optical profiling system (Wyco NT 1100, Veeco Instruments Inc.). As detailed in the encapsulation process, different silicones and pre-stretch levels were tested without noticeable impact.

Figure 6 presents the heights of the encapsulated drops as a function of time. Figure 7 presents the membrane surface after long periods of encapsulation (days to weeks).Figure 6 (a) shows that all solvents except glycerol quickly leak through the membrane. Despite the fact that encapsulated volume of glycerol shows good stability, Figure 7 (a) shows it is nonetheless diffusing through silicone and leaving micro-droplets ( $2.5 \mu \mathrm{m}$ in diameter) on the membrane outer surface. As a general trend, solvents with high vapor pressure and low viscosity seem to leak more rapidly. Figure 6 (b) shows good stability for the oils. The best performance is obtained with safflower oil. Variation in height of the encapsulated volume is only of a few percent over 20 days. Nevertheless, as for glycerol, Figure 7 (b)-(c) shows that all tested oils diffused through silicone. While it might be acceptable for some applications, the presence of liquid on the outer surface of the membrane is not acceptable for optical devices. It can indeed significantly affect the optical quality of the device by drastically increasing light scattering. Figure 6 (c) shows good stability of the silicone pre-polymers and the ionic liquid. Figure 7 (d) shows that over a period of 20 days the ionic liquid didn't diffuse through the membrane. Figure 7 (e) shows that over a period of 35 days the LSR20-B didn't diffuse through the membrane. Similar observations were done the Sylgard 184 pre-polymers. According to these results, negligible vapor pressure and high viscosity seem to be the best combination to ensure leak-free encapsulation. 


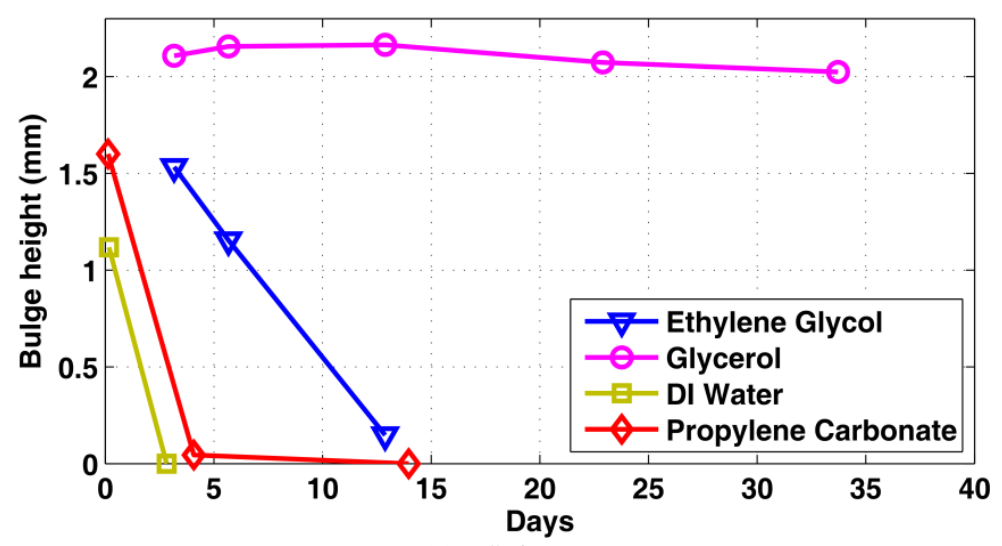

(a) Solvents

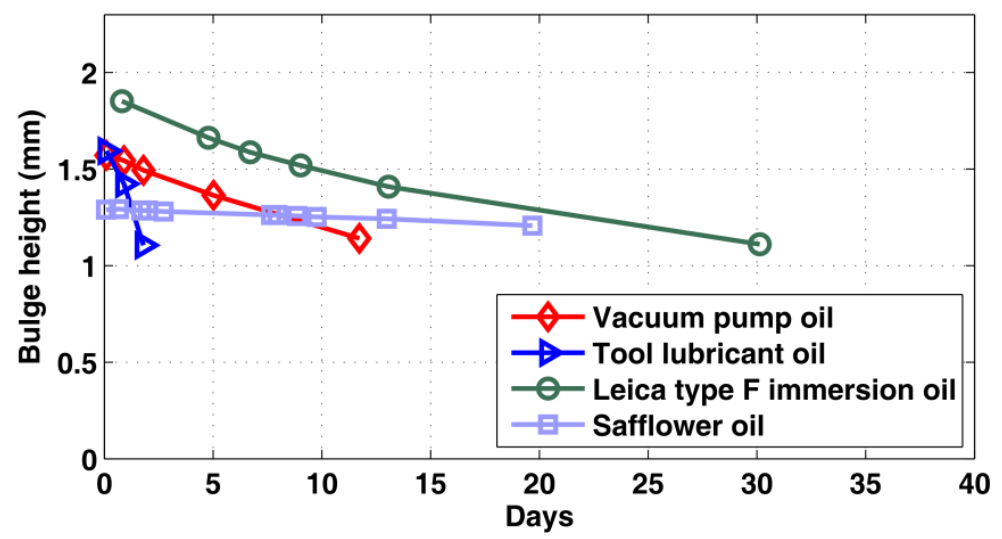

(b) Oils

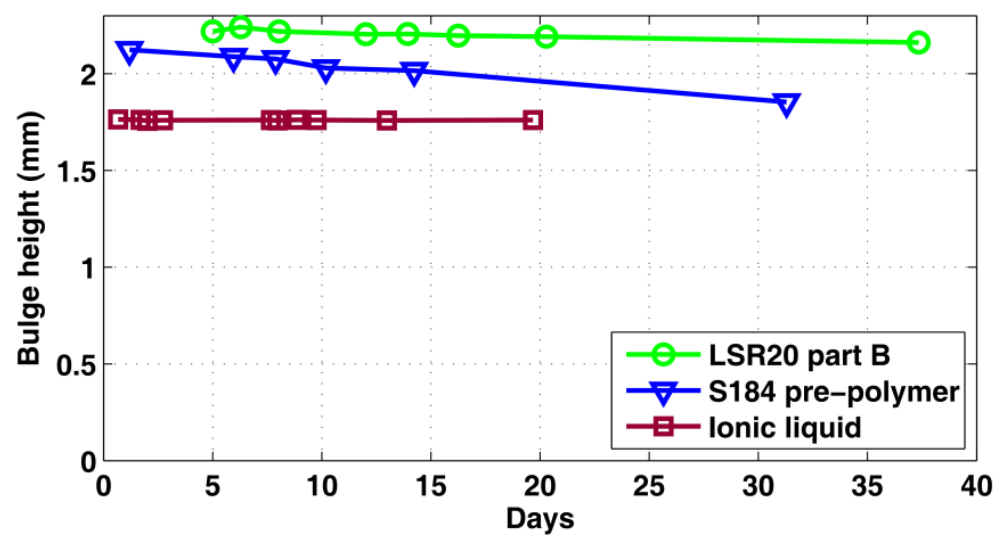

(c) Silicone pre-polymers and ionic liquid

Figure 6: Drops of liquids were encapsulated under a thin silicone elastomer membrane. The height of the encapsulated volume was monitored over time in order to track any leakage due to liquid diffusion and evaporation through the membrane. Two different silicones (CF19 and LSR4305) were tested for the encapsulation but no difference was observed. 


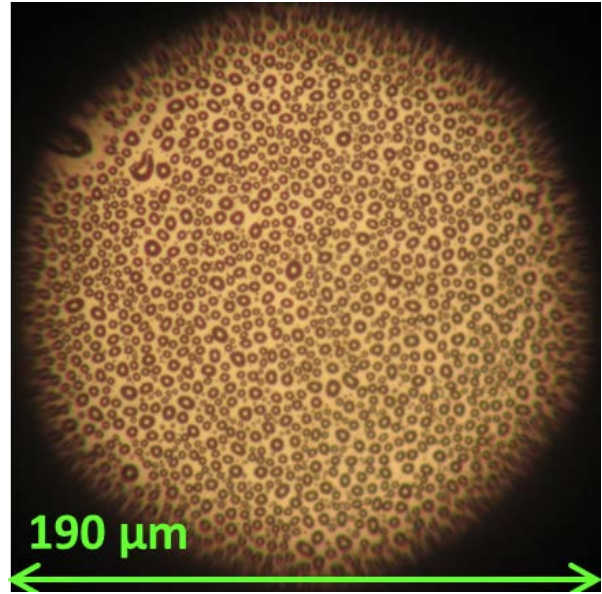

(a) Glycerol

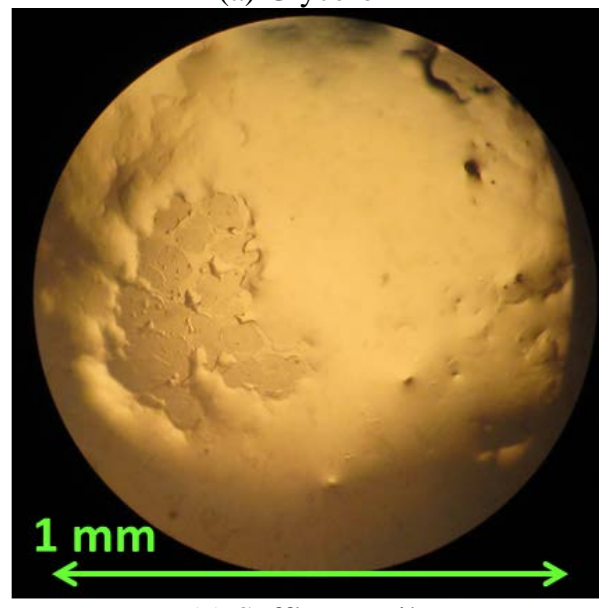

(c) Safflower oil

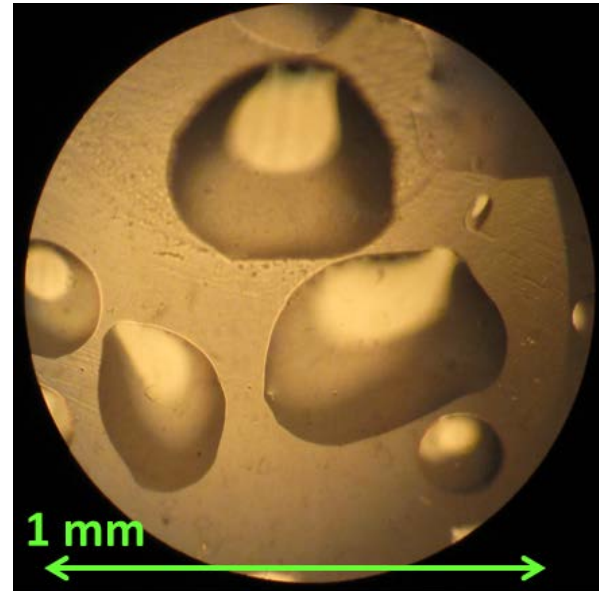

(b) Tool lubricant oil

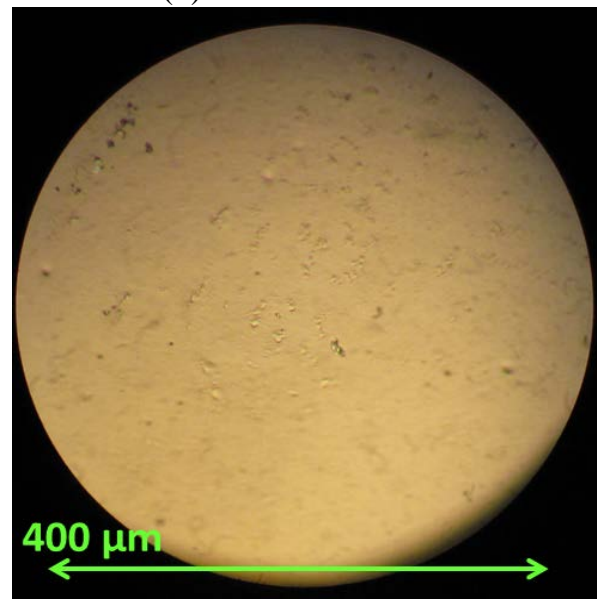

(d) Ionic liquid $\mathrm{TF}_{2} \mathrm{~N}$

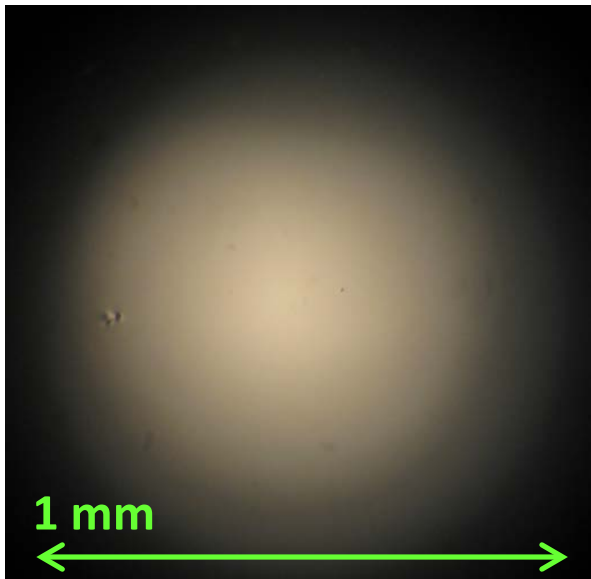

(e) Silicone pre-polymer LSR20

Figure 7: Drops of liquid were encapsulated under thin silicone membrane. The outer face of the silicone membrane was optically inspected to verify if any sign of liquid diffusion through the membrane could be observed. 


\section{OPERATION OF DEAS IN A CONDUCTIVE LIQUID}

\subsection{Design considerations}

In this section we investigate the effects of conductive immersion-liquids on DEAs. Two possible configurations are studied: single-sided and full immersion. Design considerations are discussed based on the equivalent electrical circuits.

An example of single-sided immersion is presented in Figure 8(a). Compliant electrodes are patterned on both sides of a membrane and covered by a passivation layer. The bottom side of the actuator is immersed in a conductive liquid. The passivation consists of a thin (with respect to the membrane) silicone coating which can have multiple functionalities. For instance, it can ensure electrical isolation between the electrode and its surrounding, or minimize the electric field in passive regions of DEAs immersed in conductive liquids.

Figure 8 (b) presents the equivalent electrical circuit if the liquid is at a floating potential. $\mathrm{C}_{\mathrm{DEA}}$ is the actuator's capacitance, measured between the two compliant electrodes. $\mathrm{C}_{\mathrm{P}}{ }^{1}$ and $\mathrm{C}_{\mathrm{P}}{ }^{2}$ are the parasitic capacitances of the top and bottom electrodes, respectively. Figure 8 (c) presents the equivalent electrical circuit if the liquid is grounded. As we can see, grounding the liquid effectively short-circuits $C_{P}{ }^{2}$ and leaves only one parasitic capacitance. The amplitude of $C_{P}^{1}$ is proportional to the passive area of the top electrode (i.e. not overlapping the bottom electrode), and inversely proportional to the device total thickness (membrane + passivation).

We can see from this analysis that single-sided immersion mostly affects non-overlapping regions of the electrode, which should therefore be avoided. When it is not possible, the amplitude of the parasitic actuation can be limited using a bottom passivation. A thicker passivation will decrease the parasitic electric field across the membrane and the resulting Maxwell stresses. On the other hand, it is also important to take in account the stiffening effect of the passivation. The ideal bottom passivation would be as thick and soft as possible. As for the top passivation, it has no effect on the equivalent electrical circuit and is therefore not essential on the electrical point of view.

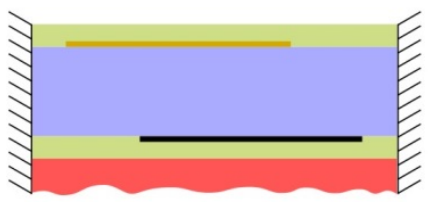

(a) Single-sided immersion

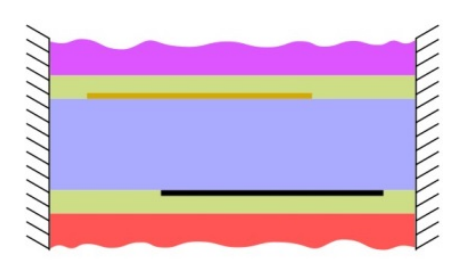

(d) Full immersion

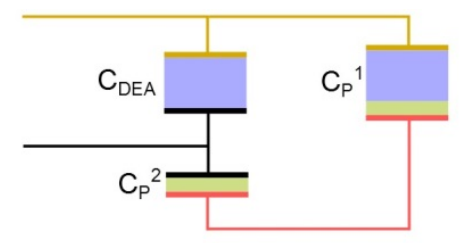

(b) Partial immersion - Floating liquid

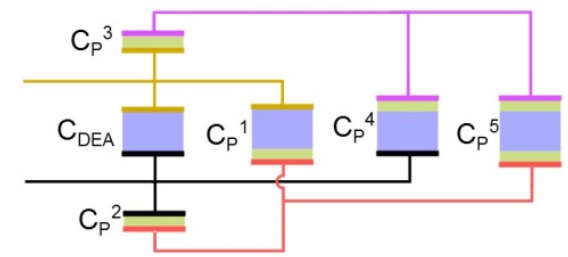

(e) Full immersion - Floating liquid

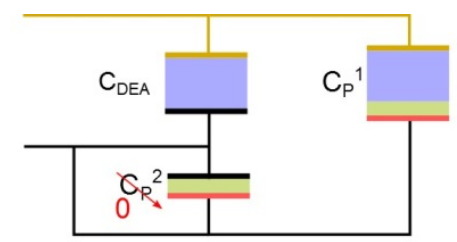

(c) Partial immersion - Grounded liquid

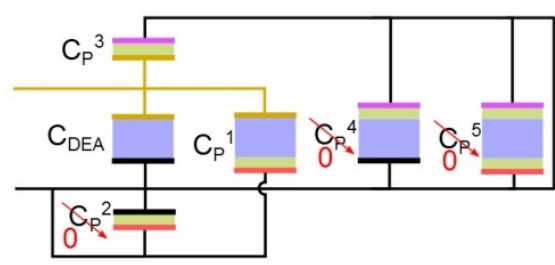

(f) Full immersion - Grounded liquid

: Active membrane $\quad$ : Passivation layer $\mathbf{\square}$ : Grounded electrode $\square$ : Live electrode $\quad Z$ : Conductive liquid

Figure 8: (a) A schematic cross-section presents the case of single-sided immersion. The equivalent electrical circuits are presented with the liquid (b) at floating potential and (c) grounded. (d) A schematic cross-section presents the case of full immersion. The equivalent electrical circuits are presented with the liquid (e) at floating potential (in this representation the top and bottom liquids are not at the same potential) and (f) grounded. 
Figure 8 (d) presents an example of full immersion where both sides of the actuator are submerged by a conductive liquid. Figure 8 (e) presents the equivalent electrical circuit if the top and bottom liquids are at different floating potentials. $C_{D E A}$ is the actuator's capacitance, measured between the two compliant electrodes. $C_{P}{ }^{1}$ and $C_{P}{ }^{3}$ are the parasitic capacitances of the top electrode. $C_{P}{ }^{2}$ and $C_{P}{ }^{4}$ are the parasitic capacitance of the bottom electrode. $C_{P}{ }^{5}$ is the parasitic capacitance of the immersion liquid.

Figure 8 (f) presents the equivalent electrical circuit if the top and bottom liquids are grounded. As we can see, grounding the liquid on both sides of the DEA effectively short-circuits $C_{P}^{2}, C_{P}^{4}$ and $C_{P}^{5}$, leaving only $C_{P}^{1}$ and $C_{P}^{3}$. The effect of $C_{P}{ }^{1}$ was already discussed for partial immersion; it induces mechanical strain in passive regions of the top electrode. As for $\mathrm{C}_{\mathrm{P}}{ }^{3}$, its amplitude is inversely proportional to the thickness of the top passivation and it induces mechanical strain in regions covered by the top electrode. In addition, if the top passivation is thinner than the active membrane, the maximum operating voltage decreases (i.e. dielectric breakdown occurs across the top passivation first).

We can see from this analysis that full immersion mostly affects the area covered by the top electrode. The bottom passivation can minimize parasitic actuation in the non-overlapping region of the electrodes. Its role has already been discussed in the case of single-sided immersion. The top passivation provides electrical isolation between the live electrode and the grounded liquid. In addition, it can minimize parasitic actuation in the area covered by the live electrode. Similar to the bottom passivation, the ideal top passivation would be as thick and soft as possible.

\subsection{Immersion of DEAs in a conductive aqueous solution}

We experimentally investigated the case of single-sided immersion of DEAs in a conductive aqueous solution. The stability in time of an immersed actuator and the possible impact of diffusion into its silicone membrane were assessed by monitoring the device actuation strain and capacitance over periodic actuation.

The device that was used for the experiment is presented in Figure 9 (a)-(b). The actuator consists of a $30 \mu \mathrm{m}$ thick silicone membrane (Sylgard 186, Dow Corning) under high uniaxial pre-stretch $\left(\lambda_{1}=2.7, \lambda_{2}=1\right)$. The membrane is sandwiched between two stretchable electrodes (in black on the picture) composed of an elastomer-carbon black composite and patterned by pad-printing ${ }^{2}$. Both electrodes are covered by a thin $(\sim 2 \mu \mathrm{m})$ passivation layer (Sylgard 184). The experiment was done under partial immersion of the DEA in order to simplify the experimental setup. The actuator was placed in an adapted mount as presented in Figure 9 (c)-(e). The mount is composed of two PMMA plates and an elastomer (LSR4305) mould. The top plate is screwed on to bottom one, pressing the assembly together thus forming a watertight chamber under the actuator. Once the device is properly mounted, the chamber can be filled with liquid. Two reservoirs on the sides are meant to compensate for the slow diffusion of liquid through the membrane. In addition, a glass plate is used to seal the top side. It creates a closed environment where the percentage of humidity quickly reaches equilibrium, therefore limiting the evaporation rate. The glass plate also serves as a protection against external contamination while maintaining optical access to the actuator.

The top electrode is connected to a high-voltage power supply whereas the bottom electrode and the liquid are grounded. Given that the electrodes are electrically isolated by the passivation layers, a metallic conductor patterned on the rigid PMMA frame is used to ground the liquid. In addition to the power supply, a capacitive sensor (Universal Transducer Interface, SMARTEC BV) is connected in parallel with the actuator. High-voltage relays are used to control which element is connected, allowing for the power supply and the capacitive sensor to be connected individually. When the assembly and the electrical connections are completed, the device is placed under a camera (uEye UI-1465LE-C, IDS Imaging Development Systems GmbH) and an optical microscope objective (Nikon L plan, 2.X/0.075). From that point, everything is controlled through a computer interface. The maximum applied voltage, the actuation frequency and the sampling periods ( $T_{\mathrm{e}}$ : actuation strain and $T_{C}$ : DEA capacitance) must be selected before starting the measurement. The strain-voltage curve of the actuator is measured every $T_{\mathrm{e}}$ cycles. It is obtained by taking pictures of the active area at increasing voltages while tracking the electrodes boundaries. Every $T_{C}$ cycles the high-voltage source is disconnected from the DEA and the capacitive sensor is connected. In addition to these two measurements, the driving current and the driving voltage are continuously monitored. 


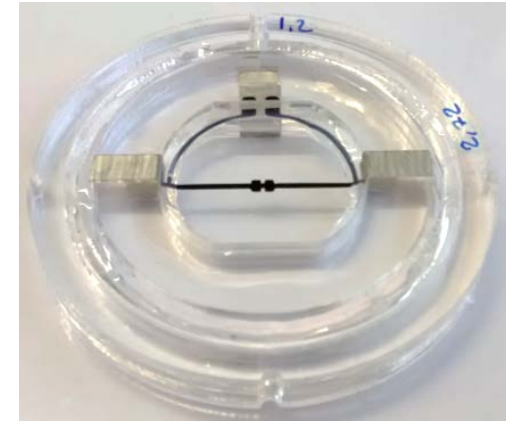

(a)

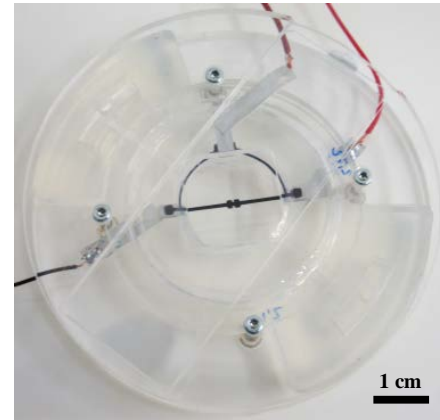

(c)

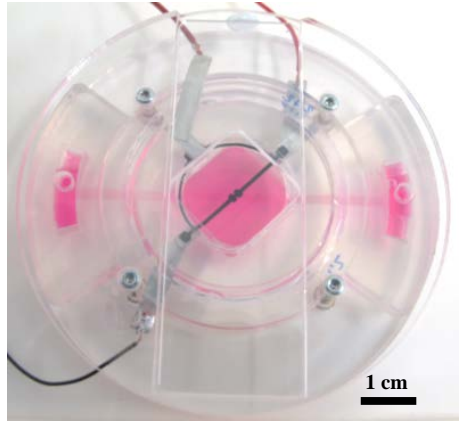

(d)

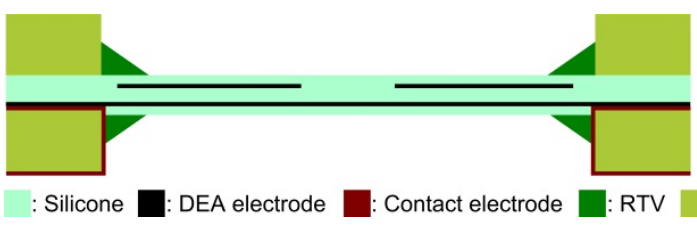

(b)

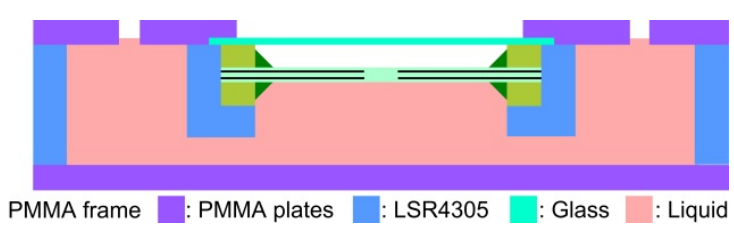

(e)

Figure 9: (a) The actuator is composed of a $30 \mu \mathrm{m}$ thick silicone (Sylgard 186) membrane under high uniaxial pre-stretch. Elastomer-carbon black composite electrodes (in black) are patterned on both sides of the membrane. (b) A schematic crosssection of the DEA shows that the electrodes are passivated with a thin $(\sim 2 \mu \mathrm{m})$ silicone (Sylgard 184) layer. (c) The device is mounted on top of an immersion chamber and connected to a high-voltage power supply. (d) The immersion chamber is filled with an aqueous solution. (e) A schematic cross-section shows that only one side of the actuator is immersed in liquid.

\subsection{Actuation strain and capacitance stability over periodic actuation}

The immersion liquid that was used for the experiment is a biological cell growth medium. It is mostly composed of water and salts. In addition, this type of solution normally includes a carbon source (ex. glucose) as well as an amino acid and nitrogen source (ex. yeast extract). The device under test was actuated at $1 \mathrm{~Hz}$ with a square wave signal (50\% duty cycles) oscillating between $0-3.5 \mathrm{kV}$. The DEA was actuated for more than 45k cycles and was still working when the experiment stopped.

Figure 10 presents the device actuation strain as a function of the number of cycles for different actuation voltages. As we can see, the un-actuated state doesn't come back to zero strain. There is a rapid increase within the first 5k cycles, after what it stays stable over the next 40k cycles. These results suggest a modification in the stress distribution across the membrane. One potential explanation is that repeated actuation is breaking some of the polymer chains in the active area, thus locally softening the membrane and breaking the force equilibrium. This occurs quickly due to the high uniaxial pre-stretch of the membrane $\left(\lambda_{1}=2.7, \lambda_{2}=1\right)$. As a result, the passive area of the pre-stretched membrane slightly stretches the active area until it reaches the new equilibrium position. This explanation is further supported by the fact that remnant strain can also be observed at higher working voltages. This is at least true over a certain number of cycles, after what other effects start masking the residual strain.

While the actuation strain at $0 \mathrm{~V}$ increases by $1 \%$ over $45 \mathrm{k}$ cycles, it decreases by about $2 \%$ at $3.5 \mathrm{kV}$. In other words, the shape of the strain-voltage curve varies with the number of cycles. This phenomenon can't be explained by the remnant strain since its effect is simply to translate the strain-voltage curve along the strain axis. One possible explanation would be a gradual degradation of the electrodes which would slowly decrease the maximum actuation strain. Experimental verification however demonstrated that the electrodes experienced little degradation. A more plausible explanation is a modification of the in-plane actuation profile. High uniaxial pre-stretch was applied on the membrane in order to achieve uniaxial actuation. The strain measurements showed in Figure 10 were made along to the high-strain axis. It is expected that gradual relaxation of stress in the membrane would decrease actuation strain ratio between high-strain and low-strain axis. It is also important to mention that similar behavior was observed on actuators operated in air, therefore excluding the immersion liquid as a potential explanation to the strain-voltage curve evolution. 


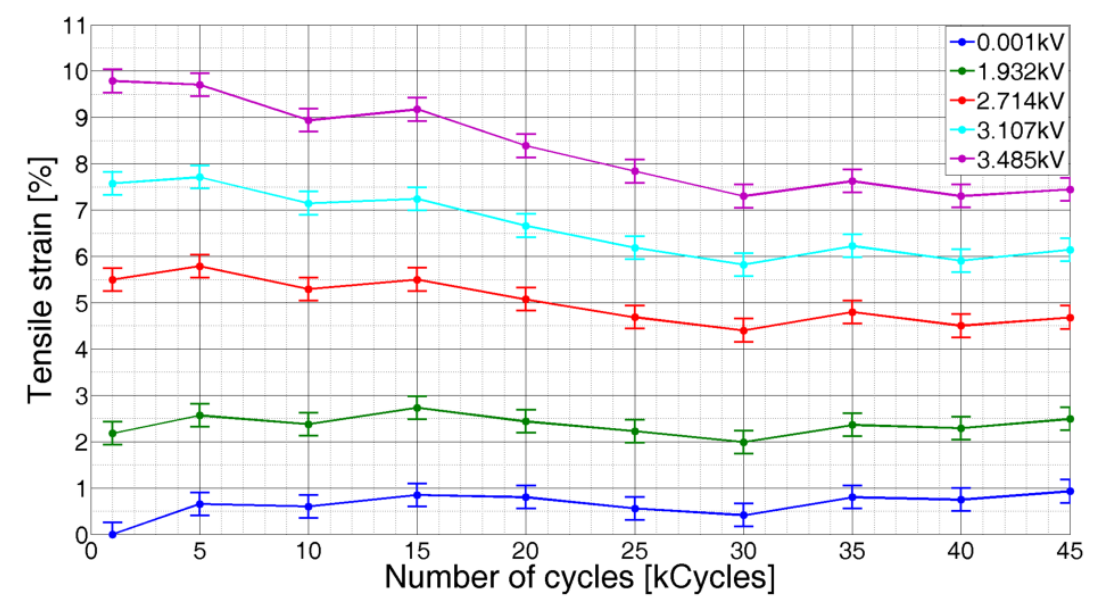

Figure 10: One side of a DEA was immersed in a conductive aqueous solution. It was actuated using a $1 \mathrm{~Hz}$ square wave signal with a $50 \%$ duty cycle. The strain-voltage curve of the actuator was measured every $5 \mathrm{k}$ cycles. The graph presents the actuation strain as a function of the number of cycles for 5 different voltages.

The actuation strain of DEAs can be affected by many parameters. While it is interesting to study its stability over periodic actuation, it doesn't give much information regarding the underlying phenomenon. In order to have a better understanding of how our actuator is affected by immersion liquids, we monitored its capacitance. The high-voltage source was periodically disconnected and the capacitance was measured. Figure 11 presents the actuator capacitance as a function of the number of cycles. The capacitance decreases continuously by about $1 \%$ over the first $5 \mathrm{k}$ cycles, and by an additional $1 \%$ in a non-continuous trend over the next $40 \mathrm{k}$ cycles. The variations observed around $40 \mathrm{k}$ cycles were caused by neon lights in the experimental room. It shows how sensitive is the setup and how small are the recorded capacitance variations.

The results presented in this section give a better understanding of how DEAs operate in conductive liquids. The actuation strain stability that was obtained is comparable to performance in air. One of the initial concerns was that the diffusion of ionic species could induce premature breakdown of the actuator. The capacitive measurements showed only negligible variations, a sign that the dimensions and the dielectric constant of the membrane are stable. Although it is not a direct measurement, these results suggest that ionic diffusion in the membrane is a negligible phenomenon. In addition, no significant effect could be observed on the life time of DEAs. Experiments were stopped due to partial evaporation of the immersion liquid.

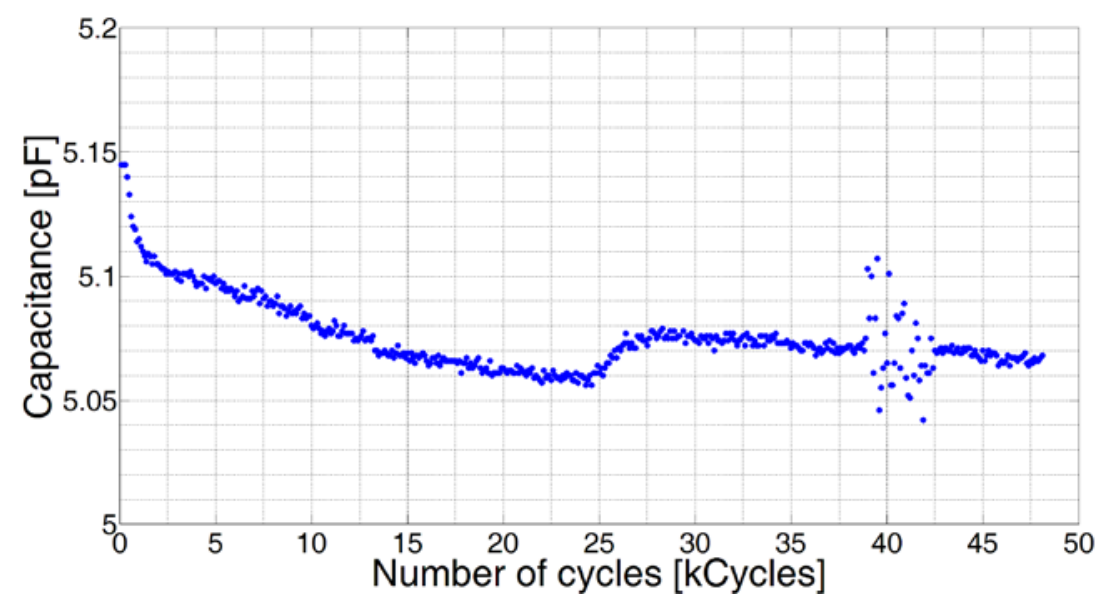

Figure 11: One side of a DEA was immersed in a conductive aqueous solution. It was actuated using a $1 \mathrm{~Hz}$ square wave signal (50\% duty cycle) oscillating between $0 \mathrm{~V}$ and $3.5 \mathrm{kV}$. The high-voltage power supply was disconnected every 100 cycles to measure the actuator capacitance. The graph presents the DEA capacitance as a function of the number of cycles. 


\section{CONCLUSION}

Interfacing DEAs with liquids is a challenging task about which only little information can be found in literature. In this work we investigated the encapsulation of liquids in thin silicone elastomer membranes. A set of 12 liquids including solvents, oils, silicone pre-polymers and one ionic liquid were experimentally tested. It was demonstrated that all solvents and oils eventually diffused through the membrane. As for the silicone pre-polymers and the ionic liquid, they all exhibited very good compatibility. No sign of diffusion through the silicone membrane was observed, even after more than one month of encapsulation (over 2 years of stability was observed for Sylgard 184 pre-polymer). The main disadvantage of pre-polymer is their high dynamic viscosity which could limit the response time of fluidically-coupled DEAs. For this type of applications, ionic liquids are an interesting alternative. In addition to their low viscosity they can be used as liquid electrodes. Their electrical properties can however make the integration in DEAs more difficult. In summary, all the tested liquids were chemically inert to silicone and negligible vapor pressure appeared to be the key parameter for encapsulation.

This work also investigated the actuation of DEAs in conductive liquids. The analysis of the equivalent electrical circuits showed that immersion can significantly impact the actuation performance. One conclusion is that non-overlapping regions of the electrodes should be minimized. Otherwise, significant mechanical strain can be induced in passive regions of the device. In order to experimentally assess the effects of conductive liquids, the actuation strain and the capacitance of a DEA were monitored over periodic actuation. The device was operated over more than 45k cycles and showed actuation strain stability comparable to performance in air. In addition, the capacitance measurements varied by only $2 \%$, demonstrating that diffusion of foreign particles in the membrane is negligible. The experimental results presented in this work show that the actuation strain stability and the lifetime of DEAs is not (significantly) affected by the immersion in conductive aqueous solutions.

\section{ACKNOLEDGEMENTS}

This research was supported by the Swiss National Science Foundation (SNSF) under Grant No. 200020_153122 and the SNSF R'equip program 206021_139187.

\section{REFERENCES}

[1] Keplinger, C., Li, T., Baumgartner, R., Suo, Z.., Bauer, S., "Harnessing snap-through instability in soft dielectrics to achieve giant voltage-triggered deformation,” Soft Matter 8(2), 285 (2012).

[2] Maffli, L., Rosset, S., Ghilardi, M., Carpi, F.., Shea, H., "Ultrafast All-Polymer Electrically Tunable Silicone Lenses,” Adv. Funct. Mater., n/a - n/a (2015).

[3] Goulbourne, N. C., Frecker, M. I.., Mockensturm, E., "Electro-elastic modeling of a dielectric elastomer diaphragm for a prostetic blood pump,” Proc. SPIE 5385, 122-133 (2004).

[4] Lotz, P., Matysek, M.., Schlaak, H. F., "Peristaltic pump made of dielectric elastomer actuators,” Proc. SPIE 7287, 72872D (2009).

[5] Pelrine, R., Sommer-Larsen, P., Kornbluh, R. D., Heydt, R., Kofod, G., Pei, Q.., Gravesen, P., “Applications of dielectric elastomer actuators," Proc. SPIE 4329, 335-349 (2001).

[6] Loverich, J. J., Kanno, I.., Kotera, H., “Concepts for a new class of all-polymer micropumps.,” Lab Chip 6(9), 1147-1154, Royal Society of Chemistry (2006).

[7] Maffli, L., O’Brien, B., Rosset, S.., Shea, H., “Pump it up,” Proc. SPIE 8340, 83402Q - 1-83402Q - 16 (2012). 
[8] Carpi, F., Frediani, G., Turco, S.., De Rossi, D., "Bioinspired Tunable Lens with Muscle-Like Electroactive Elastomers,” Adv. Funct. Mater. 21(21), 4152-4158 (2011).

[9] Shian, S., Diebold, R. M.., Clarke, D. R., "High-speed, compact, adaptive lenses using in-line transparent dielectric elastomer actuator membranes,” Proc. SPIE 8684, 86872D (2013).

[10] Poulin, A., Rosset, S.., Shea, H., “Toward compression of small cell population: harnessing stress in passive regions of dielectric elastomer actuators,” SPIE Smart Struct. Mater. + Nondestruct. Eval. Heal. Monit., Y. BarCohen, Ed., 90561Q, International Society for Optics and Photonics (2014).

[11] Akbari, S.., Shea, H. R., "An array of $100 \mu \mathrm{m} \times 100 \mu \mathrm{m}$ dielectric elastomer actuators with $80 \%$ strain for tissue engineering applications,” Sensors Actuators A Phys. 186, 236-241, Elsevier B.V. (2012).

[12] Friese, C., Werber, A., Krogmann, F., Mönch, W.., Zappe, H., "Materials, effects and components for tunable micro-optics,” IEEJ Trans. Electr. Electron. Eng. 2(3), 232-248 (2007).

[13] Werber, A.., Zappe, H., “Tunable, membrane-based, liquid-filled micro-lenses,” 13th Int. Conf. Solid-State Sensors, Actuators Microsystems, 2005. Dig. Tech. Pap. TRANSDUCERS ’05. 1, 1018-1021, IEEE.

[14] Carpi, F., Frediani, G.., De Rossi, D., "Hydrostatically Coupled Dielectric Elastomer Actuators,” IEEE/ASME Trans. Mechatronics 15(2), 308-315 (2010).

[15] Carpi, F., Frediani, G., Tarantino, S.., De Rossi, D., "Millimetre-scale bubble-like dielectric elastomer actuators,” Polym. Int. 59(3), 407-414 (2009).

[16] Carpi, F., Frediani, G., Nanni, M.., Rossi, D. De., “Granularly Coupled Dielectric Elastomer Actuators,” IEEE/ASME Trans. Mechatronics 16(1), 16-23, IEEE (2011).

[17] Lee, J. N., Park, C.., Whitesides, G. M., "Solvent compatibility of poly(dimethylsiloxane)-based microfluidic devices.,” Anal. Chem. 75(23), 6544-6554 (2003).

[18] Akbari, S., “Arrays of dielectric elastomer microactuators for cell mechanotransduction,” 134, Ecole Polytechnqiue Fédérale de Lausanne (2013). doi:10.5075/epfl-thesis-5697

[19] Maffli, L., "Fluidically-coupled dielectric elastomer actuator structures for tunable optics and microfluidics,” 187, Ecole Polytechnique Fédérale de Lausanne (2014). doi:10.5075/epfl-thesis-6305 